\title{
EDITORIAL
}

\section{Moving toward a unified effort to understand the nature and causes of language disorders}

\author{
MABEL L. RICE and STEVEN F. WARREN \\ University of Kansas
}

\begin{abstract}
ADDRESS FOR CORRESPONDENCE
Mabel L. Rice, University of Kansas, Child Language Doctoral Program, 1000 Sunnyside Avenue, 3031 Dole Center, Lawrence, KS 66045-7555. E-mail: mabel@ku.edu
\end{abstract}

The nature and causes of language disorders are research topics familiar to the readership of Applied Psycholinguistics. Current inquiries place these topics in a dynamic and sprawling multidisciplinary context manifested through investigations of linguistic acquisition, cognitive development, genetics, neurocortical processes, cognitive neurolinguistics, behavioral phenotypes, and language intervention. This list is not complete by any means, but it does suggest the wide front of the current search for better knowledge about what causes language disorders and the dimensions of language acquisition that are affected. Although there is considerable momentum underway in current inquiries, the full potential for scientific advancement is hampered by fragmentation in the field, in part attributable to partitioning by diagnostic categories of affectedness. Investigators (and their funding sources) often focus on a particular clinical group, such as specific language impairment (SLI), autism/autism spectrum disorders (ASD), Williams syndrome (WS), Down syndrome (DS), or fragile X syndrome (FXS). Although this is not exclusively the case, it is relatively difficult to carry out comparative studies across the multiple clinical conditions in which language disorders appear and to monitor developments across such a wide front of investigation. The consequence is that findings are distributed across different publication outlets and different groups of scholars, a situation that can limit our appreciation of the ways in which language disorders are manifest, and the identification of common etiological factors.

The papers in this special issue are the products of an effort to establish connections among scholars encompassing a wide range of expertise relevant to language disorders. The intent is to move toward a more unified effort to understand the nature and causes of language disorders. The papers are unusual in several ways. Instead of the typical reports of individual studies or the work of particular authors, these papers draw upon the outcomes of two previous meetings of a group of scholars. The scholars carry out programmatic research in language disorders in one or more clinical groups and represent a range of expertise, including behavioral

(C) 2005 Cambridge University Press 0142-7164/05 \$12.00 
Rice \& Warren: Unifying the effort to understand language disorders

measurement of language abilities, cognitive abilities, neurocortical processes, genetics, and language intervention. The group was initially brought together for the purpose of sharing information about current investigations, identifying converging outcomes and common scientific gaps or challenges, and suggesting new directions of research. This meeting, sponsored by the Merrill Advanced Studies Center of the University of Kansas, was held in Tempe, Arizona, in May 2002. The participants, who are listed here in alphabetical order, are Leonard Abbeduto, Donald Bailey, Judith Cooper, Catherine Lord, Peggy McCardle, Carolyn Mervis, Colleen Morris, Ralph-Axel Müller, Colin Phillips, Mabel Rice, Shelley Smith, Helen Tager-Flusberg, Bruce Tomblin, Steve Warren, and Andrea Zukowski. Papers from that conference appear in Rice and Warren (2004).

The group reconvened in September 2003 under sponsorship by the National Institute of Child Health and Human Development (NICHD) and the Merrill Advanced Studies Center, for the purpose of moving the discussions toward the development of position papers about what is needed in future research. The group was joined by Simon Fisher, who provided a keynote address about his work on the FOXP2 gene. The group worked collaboratively on the following topics: research design, measurement issues, neurocognitive studies, genetics studies (including neuromolecular studies), and next steps in the studies of genetics and language.

The set of papers in this Special Edition are the products of those discussions. Thus, this collection of papers is something akin to state of the art papers from a small group of highly seasoned experts, with an eye toward future directions of investigation, based on emerging generalizations from current studies of language disorders, neurocortical processes, genetics, and neuromolecular genetics. The papers were not vetted through a formal position paper process. Instead, authors were nominated/selected out of the group discussions and are expressing their individual perspectives on the topics represented, in addition to summarizing key elements of the group's discussions.

The papers can be briefly summarized as follows. The opening paper, by Rice, Warren, and Betz, provides an overview of the language symptoms of the clinical disorders represented in the volume: ASD, DS, FXS, SLI, and WS. This overview draws heavily on the content of individual chapters in Rice and Warren (2004). The clinical groups selected for description correspond to the clinical groups studied by the experts who participated in the discussions. This is not an exhaustive list of diagnostic categories associated with language disorders; it is, instead, a diverse set of representative clinical conditions for which there is currently strong programmatic research underway in the area of gene/environment effects. Another caveat is that the summary paper provided here is not intended to be an exhaustive review of the research literature on language disorders. Instead, it is meant to serve as a background for the other papers, to highlight key points of comparison, to provide an entry point into the current diverse literature, and to call for more systematic comparative investigations of the symptoms of language disorders.

The second paper, authored by Helen Tager-Flusberg, addresses the important topic of research design. She addresses key questions pertaining to the language phenotypes that characterize specific disorders, key precursors and predictors of language acquisition in developmental language disorders, the genes that contribute to developmental language disorders in different syndromes, and the 
Rice \& Warren: Unifying the effort to understand language disorders

environmental factors that influence the trajectories of language acquisition in developmental language disorders. She identifies future directions and challenges that need to be addressed.

In the third paper, Carolyn Mervis and Byron Robinson address the measurement issues relevant to genotype/phenotype research and profiling. In particular, they examine the challenges posed by the need to establish language and associated cognitive profiles of syndromes with diverse genotypes. They identify six central issues and provide a close examination of the potential measurement pitfalls. They suggest ways to guard against measurement error in current studies and call for more robust instruments with more extensive normative data on which to base phenotype descriptions in future studies.

The next two papers provide contrasting perspectives on neurocortical studies. Ralph-Axel Müller calls for a bottom-up approach, in which the etiological pathways for language disorders are examined by beginning with underlying "elemental developmental disturbances" in the early-emerging sensorimotor impairments. Colin Phillips puts the case for a top-down approach, focusing on the language phenotypes of different clinical groups and the ways in which electrophysiological brain measures can clarify language phenotypes. There is no dispute about the need for both kinds of neurocortical studies. It is agreed that in an ideal, broadly based program of research, both approaches would be employed. These two papers provide thoughtful analyses of the rationale and assumptions for each perspective, the methods involved, and the ways in which new information can be especially informative.

Shelley Smith and Colleen Morris provide a succinct and clear-minded synthesis of the state of the art in research on the relationship of genes, environments, and developmental language disorders, with an eye toward the next generation of studies. In the following paper, Simon Fisher narrows the genetics focus to the level of molecular mechanisms underlying speech and language disorders. This pair of papers makes it very clear that the momentum of genetics inquiry is encouraging. At the same time, the business of sorting out the genetics of language disorders and the related system of higher cognitive mechanisms is shaping up as challenging, if not downright daunting, as studies of human genetics move forward. As we learn more, we have a more complete appreciation of the complexities involved.

The final paper in the collection, by Peggy McCardle, Judith Cooper, and Lisa Freund, brings the perspective of highly experienced research administrators at the US National Institutes of Health (NIH). They lay out next steps for research, with a focus on the development of detailed, useful behavioral phenotypes of language disorders and moving forward in the study of the relationships among genes and environment in the causes, maintenance, and remediation of language disorders. They identify key directions and key issues for investigators to consider and highlight the need for collaborative, longitudinal inquiry that bridges studies of clinical groups across the age span.

Collectively, this set of papers pulls together a wide swath of the available scientific information about language disorders, with the aim of identifying the foundation stones of future programmatic investigation that is likely to move us ahead in our understanding of the ways in which language acquisition can be vulnerable as well as robust. A wide range of scientific talent is needed for the task; we feel 
Rice \& Warren: Unifying the effort to understand language disorders

that the articles in this Special Issue serve as a model for the appropriate scope and depth of collective expertise to bring to future studies.

\section{ACKNOWLEDGMENTS}

We acknowledge the support of the Merrill Advanced Studies Center at the University of Kansas and the NICHD. The preparation of this paper was supported by grants from the NIH to the University of Kansas through the Mental Retardation and Developmental Disabilities Research Center (P30HD002528) and the Center for Biobehavioral Neurosciences in Communication Disorders (P30DC005803). We also acknowledge the support provided by R01 grants from the NIH (DC001803, DC005526, HD003110, and HD044868).

\section{REFERENCE}

Rice, M. L., \& Warren, S. F. (2004). Developmental language disorders: From phenotypes to etiologies. Mahwah, NJ: Erlbaum. 